\title{
Delayed splenic abscess after blunt abdominal trauma in the 24- year-old woman: diagnostic difficulties and dilemma
}

\author{
ShokouhTaghipour zahir ${ }^{1}$ Koorosh Rahmani ${ }^{2}$ Saeed $\operatorname{Kargar}^{3}$ Zahra Kargar $^{4}$ \\ ${ }^{I}$ Department of pathology, Faculty of medicine, ShahidSadoughi University of Medical sciences, Yazd, Iran \\ ${ }^{2}$ Department of pathology, ShahidSadoughi University of Medical sciences, Yazd, Iran \\ ${ }^{3}$ Department of surgery, Faculty of medicine, ShahidSadoughi University of Medical sciences, Yazd, Iran \\ ${ }^{4}$ Department of pathology, ShahidSadoughi University of Medical sciences, Yazd, Iran
}

\begin{abstract}
Primary splenic abscess with unknown origin is rare and it could be mistaken with other conditions. We present a 24-year-old woman with abdominal pain and weight loss without fever or related symptoms. In ultrasound examination ill-defined anechoic mass-like lesion was evident, located near the splenic vein in retroperitoneum with spleno-systemic shunt. Patient underwent laparoscopy for ruling out malignancy but severe inflammation and adhesion-bands prevent further laparoscopic exploration. One week later patient's condition was deteriorated and she underwent second surgical exploration. In laparotomy spleen was surrounded by an abscess wall and splenectomy was done. Histopathological examination revealed subcapsular splenic hemorrhage with abscess formation.
\end{abstract}

Keywords: spleen, trauma, abscess, subcapsular

\section{Introduction}

Splenic abscess is a rare condition that could be seen in both immunocompetent and immunosuppressed patients with different etiologies $[1,2]$. The most common route of splenic infection is hematogenous spreading from an infective focus elsewhere in the body. Infective endocarditis, Typhoid fever, Paratyphoid, Malaria, Urinary tract infection and Pelvic infection are some of the common sources of bacteremia and splenic abscess formation [3]. Splenic abscess secondary to blunt trauma is a very rare condition that could mimic other diseases and late diagnosis causes high mortality rate[1,3]. Herein, we present a young female patient with vague abdominal pain and weight loss since two months ago prior to admission, however, the abdominal CT scan and ultrasound findings had not detected the splenic subcapsular hemorrhage and abscess formation. Inability to make a definitive diagnosis made the patient to undergo surgery two times and being hospitalized for a long time.

\section{Case Presentation}

A 24 -year- old woman with history of weight loss and abdominal pain since 2 months prior to admission was referred to Gastroenterology ward. In her past medical history, nine weeks prior to admission she had car accident with blunt trauma to her abdominal left upper quadrant without any other sign or symptoms. In physical examination, tenderness in upper left quadrant of abdomen was tangible. She underwent upper gastrointestinal (GI) tract endoscopy and no significant findings were reported. In ultrasound examination aggregation of omentum in the left upper quadrant of abdomen was evident with venous congestion and also colonic inflammation. Ultrasound findings were suggestive of colitis or diverticulitis (fig- 1).Spiral abdominopelvic computed tomography (CT) scan showed mass-like lesion with diameters of $43 \times 56 \mathrm{~mm}$ adjacent to the splenic vein with ill-defined borders and unclear margins that seems located in the retroperitoneum near the upper posterior part of pancreas body. Dilated veins in the left abdomen was in favour of spleno-systemic shunt. Cystic area in the left abdomen and posterior of gastric body was reported. Inflammation was evident around the mass and it seems the lesion has caused splenic vein thrombosis with shunt (fig- 2A\& 2B). The clinical differential diagnosis was GI perforation, neoplastic mass, pancreatic pseudocyst with venous thrombosis or chronic inflammation. However, the pancreas, spleen and kidneys were reported normal in ultrasound examination. She underwent laparoscopic exploration, but due to severe inflammation and adhesion-bands, only few biopsies from omentum and surrounding pancreas were taken. Histopathological findings of tissue biopsies revealed fibrofatty connective tissues with infiltration of inflammatory cells mostly neutrophils and lymphoplasma cells without any malignant cells. She was given intravenous antibiotics. Second ultrasound and radiographic findings after the first laparoscopy revealed moderate pleural effusion in the left side and small amount of pleural effusion in the right side. Passive atelectasis in the lower lobe of left lung with irregular mild enlargement of the head and body of pancreas were also seen which was suggestive of pancreatitis. However, in lab tests Amylase was not increased (table1).Oval shaped, mixed density collection in the lateral aspect of spleen was reported which caused anterior and medial displacement of spleen that could be related to pancreatic 
pseudo-cyst formation. Size of spleen and liver were normal. Air flecks in the common bile duct (CBD) with inferior displacement of the left kidney were reported. Free peritoneal fluid in the pelvis was demonstrated. One week later patient's condition was worsening. Repeat ultrasound revealed distention of abdomen with abundant gas inside of it. The Spleen size was 130X65X50 mm which was within upper limit of normal. One anechoic lesion $15 \times 9 \mathrm{~mm}$ was seen adjacent to the inferior pole of spleen and also in right subdiaphragmatic area. The Radiologist suggestion was hematoma due to the biopsy or collection of fluid. She underwent laparotomy and in surgical exploration there was an abscess in the sub-diaphragmatic area and spleen was inside the abscess, so splenectomy was done. Histopathological finding was subcapsularhaemorrhage with necrosis and severe infiltration of inflammatory cells mostly neutrophils and lymphoplasma cells (abscess formation) (fig- 3). In differential diagnosis pancreatic pseudocyst, splenic vein thrombosis, GI tract perforation, adenopathy, chronic inflammatory lesion, diverculitis and malignant lymphoma were considered. After splenectomy, patient had given intravenous broad-spectrum antibiotics for two weeks.Patient was discharged from hospital with good general condition.

\section{Discussion}

Splenic abscess formation is a rare condition that has been reported $0.05-0.7 \%$ in different population[1]. Many aetiologies were considered for splenic abscess formation such as haematogenous spreading from other infected areas in the body (pelvis, lungs, urinary tract, even mastoid and ears, also pancreatic and retroperitoneal abscess, diverticulitis and etc.) [3, 4]. Patients with history of infective endocarditis, intravenous (IV) drug abusers and immunosuppressed patients are also susceptible to splenic abscess formation. Splenic blunt trauma as a predisposing factor for splenic abscess has been also reported [4, 5]. Our patient had not had any history of infectious source in her past medical history except blunt trauma to her left upper quadrant without clinically presenting at admission. The most clinical presentation of patients with splenic abscess is a classical triad of fever, left upper quadrant pain and splenomegaly[6]. Kumar et al reported a case of splenic abscess in a healthy boy after abdominal blunt trauma who presented with fever and ultrasound detected the splenic abscess, however our patient had not had fever and ultrasound findings were not suggestive of splenic abscess [7]. Also, in our patient in the first and second ultrasound examination no increasing in size of spleen was detected. For making the correct diagnosis of splenic abscess taking a good history and performing physical examination are necessary but not sufficient and reliable, however they certainly help for differential diagnosis. Elevated left hemi-diaphragm, pleural effusion and abnormal chest radiographs were reported in literature, but in our patient in the first chest X-ray no pleural effusion or elevation of diaphragm were reported [6, 8]. Plain Abdominal X-ray could demonstrate gas collection in the left upper quadrant, as in our patient this finding was reported after first laparoscopy. Ultrasound findings are non-specific and not reliable and they're operator-dependent [6].CT scan could help in making the correct diagnosis of splenic abscess and nowadays is a standard choice for detection of splenic abscess, but in our patient it couldn't help us for definitive diagnosis and to avoid laparotomy[4,5]. The size and topography of abscess and primary disorder as well as patient's condition could affect the treatment plan. Percutaneous drainage has been suggested as the first line of treatment to avoid splenectomy [6]. Although multilocular abscesses, necrotic debris and illdefined cavities seems to be not responsive to percutaneous drainage and surgery intervention is necessary [610].In our patient ambiguity in the accurate detection of splenic abscess leads to early surgery intervention. Open splenectomy is a standard choice of treatment of splenic abscess with a mortality rate of $0-17 \%$, but laparoscopic splenectomy is also a safe method in selected patients [5,6]. After splenectomy, treatment by broad-spectrum antibiotics is effective for full recovery from the disease $[6,8]$.

\section{References}

[1] Ghidirim G, Rojnoveanu G, Mişin I, Gagauz I, Gurghiş R. [Splenicabscess--etiologic, clinical and diagnostic features].Chirurgia (Bucur). 2007 May-Jun;102(3):309-14

[2] Ferraioli G, Brunetti E, Gulizia R, Mariani G, Marone P, Filice C. Management of splenic abscess: report on 16 cases from a single center.Int J Infect Dis. 2009 Jul;13(4):524-30.

[3] Alvi AR, Kulsoom S, Shamsi G. Splenic abscess: outcome and prognostic factors. J Coll Physicians Surg Pak. 2008 Dec. 18(12):740-3.

[4] Carbonell AM, Kercher KW, Matthews BD, Joels CS, Sing RF, Heniford BT. Laparoscopic splenectomy for splenic abscess. SurgLaparoscEndoscPercutan Tech. 2004 Oct. 14(5):289-91.

[5] Iñiguez A, Butte JM, Zuñiga JM, Torres J, Llanos O. [Splenic abscesses.Report of seven cases]. Rev Med Chil. 2008 Jan;136(1):3843.

[6] Fotiadis C, Lavranos G, Patapis P, et al. Abscesses of the spleen: report of three cases. World J Gastroenterol. 2008 May 21. 14(19):3088-91

[7] Kumar A, Kumar A, Chaudhary D. Splenic abscess following blunt abdominal trauma.The Indian Journal of Pediatrics. September 1995. 62(5): 623-625

[8] Tung CC, Chen FC, Lo CJ. Splenic abscess: an easily overlooked disease?. Am Surg. 2006 Apr. 72(4):322-5

[9] Ulhaci N, Meteoglu I, Kacar F, Ozbas S. Abscess of the spleen. PatholOncol Res. 2004. 10(4):234-6

[10] Villamil-Cajoto I, Lado FL, Van den Eynde-Collado A, Díaz-Peromingo JA. [Splenic abscess: presentation of nine cases.]. Rev ChilenaInfectol. 2006 Jun. 23(2):150- 
Fig-1: Aggregation of omentum in the left upper quadrant of abdomen with venous congestion and also colonic inflammation.

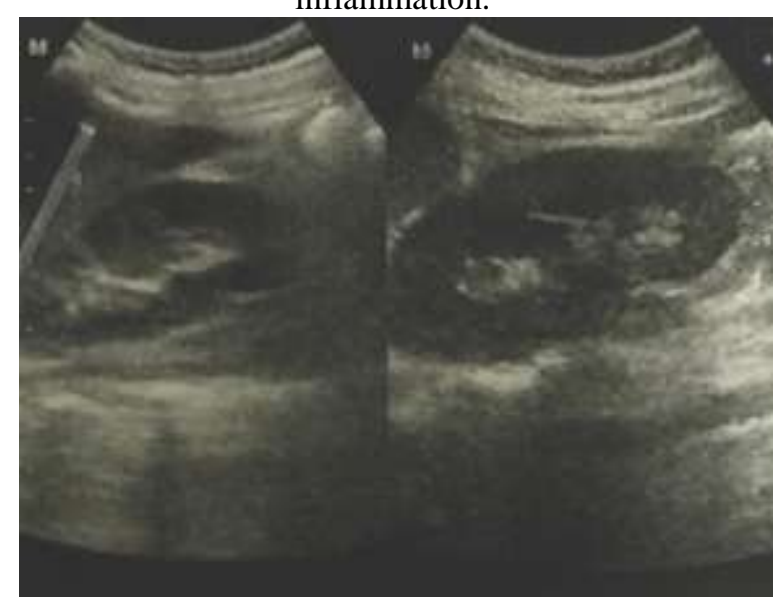

Fig- 2A: Spiral abdomino-pelvic CT scan showed mass-like lesion adjacent to the splenic vein with ill-defined borders and unclear margins.

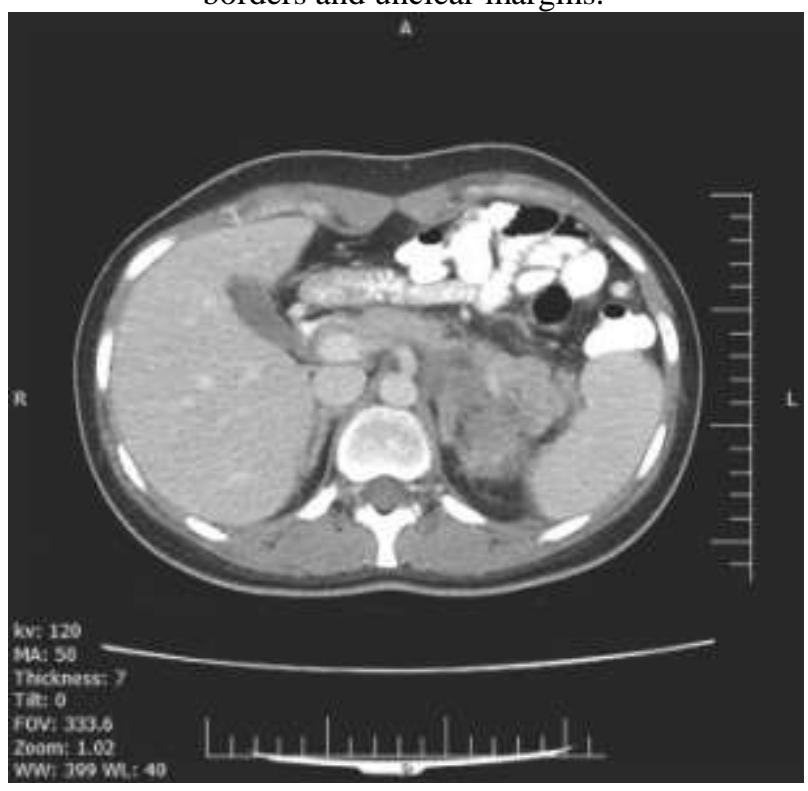

Fig- 2B: Dilated veins in the left abdomen suggested of spleno-systemic shunt with cystic area in the left abdomen and posterior of gastric body.

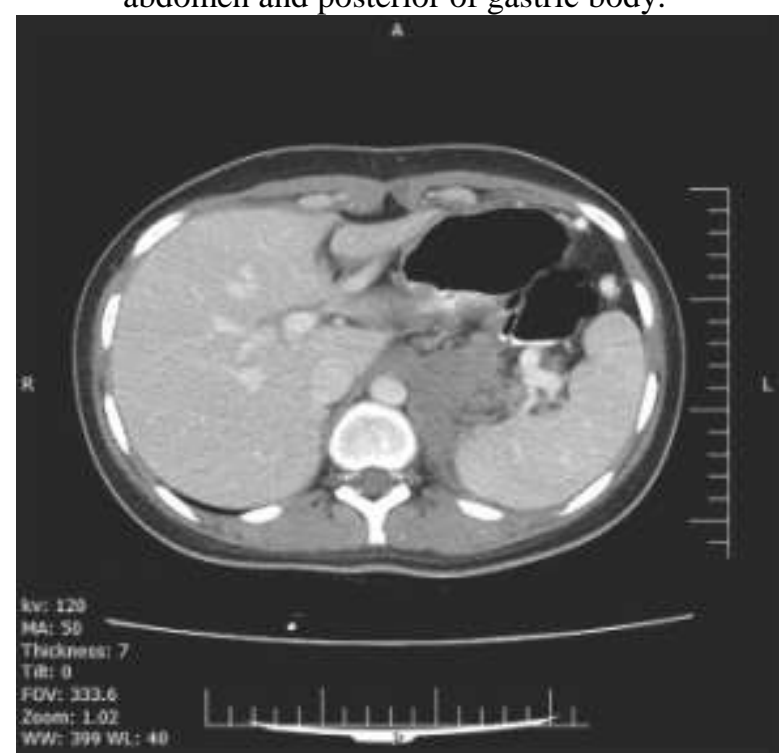


Fig- 3: Sub capsular haemorrhage with necrosis and severe infiltration of inflammatory cells mostly neutrophils and lymphoplasma cells (abscess formation) (H\&E staining x 20).

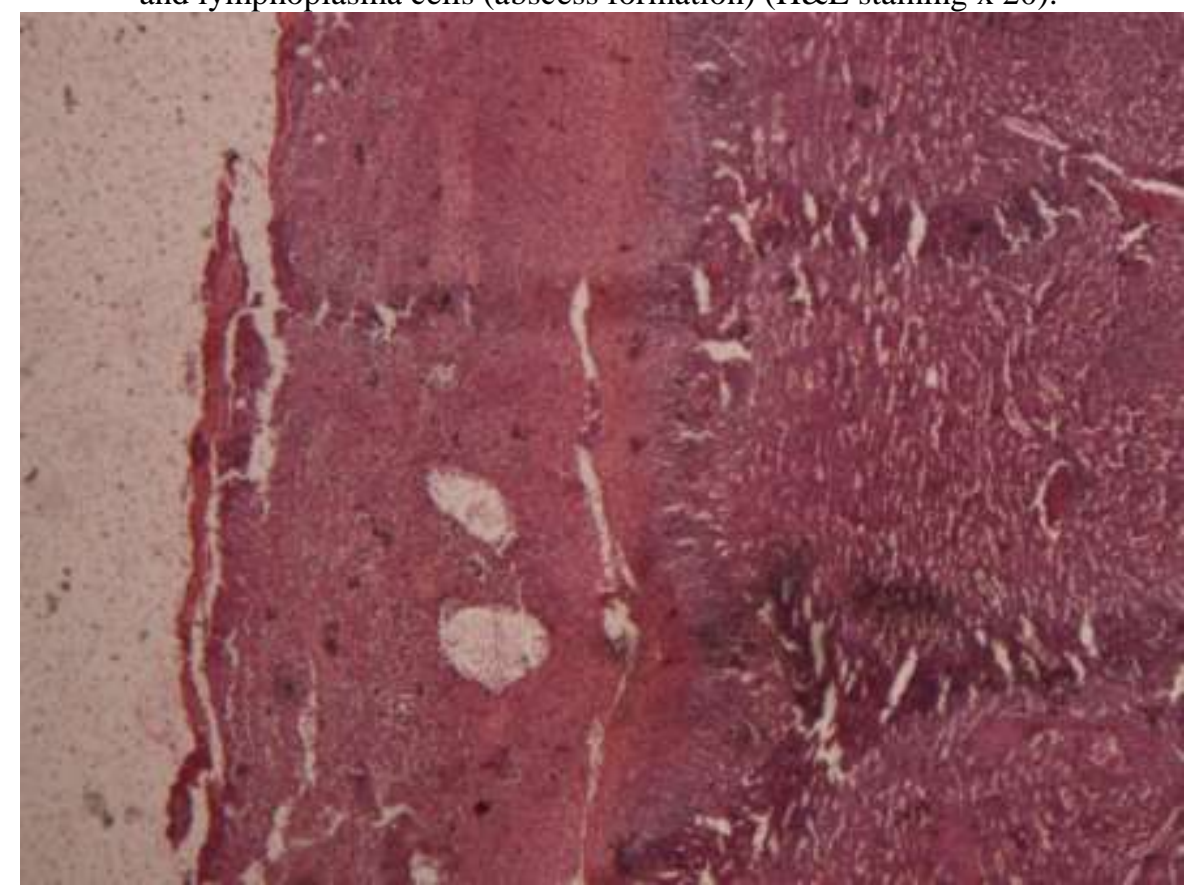

Table1: Patient's laboratory test results at the beginning of admission period.

\begin{tabular}{|l|l|l|l|}
\hline Biochemistry & Result & Unit & Normal range \\
\hline AST & 15 & IU/L & $0-40$ \\
\hline ALT & 13 & IU/L & $0-49$ \\
\hline Alkaline phosphatase & 146 & IU/L & $80-306$ \\
\hline Amylase & 35 & IU/L & Serum: $<100 \quad$ Urine: $<490$ \\
\hline Lipase & 30 & IU/L & Up to 60 \\
\hline Serology & & & \\
\hline CRP & Negative & & \\
\hline Haematology & & & \\
\hline ESR 1 hrs & 50 & $\mathrm{~mm} / \mathrm{hr}$ & $<20$ \\
\hline WBC & 9.9 & $1000 / \mathrm{UL}$ & $4-10$ \\
\hline RBC & 3.74 & $\mathrm{ml} / \mathrm{UL}$ & $4.2-5.4$ \\
\hline Hg & 9.4 & gr/dl & $13-18$ \\
\hline HCT & 30.4 & $\%$ & $36-46$ \\
\hline MCV & 81.3 & $\mathrm{Fl}$ & $77-97$ \\
\hline MCH & 25.1 & Pg & $26-32$ \\
\hline MCHC & 30.9 & gr/dl & $32-36$ \\
\hline RDW & 12.7 & $\%$ & $10.4-14.6$ \\
\hline PLT & 159 & $1000 / \mathrm{UL}$ & $145-450$ \\
\hline Neutrophil & 85 & $\%$ & \\
\hline Lymphocyte & 11 & $\%$ & \\
\hline Monocyte & 3 & $\%$ & \\
\hline Eosinophil & 1 & $\%$ & \\
\hline
\end{tabular}

